\title{
Optimizing Resource Allocation for 6G NOMA-enabled Cooperative Vehicular Networks
}

\author{
Zain Ali, Wali Ullah Khan, Member, IEEE, Asim Ihsan, Omer Waqar, \\ Guftaar Ahmad Sardar Sidhu, and Neeraj Kumar, Senior Member, IEEE,
}

\begin{abstract}
In recent years, the concept of non-orthogonal multiple access (NOMA) has gathered much attention due to its potential to offer high spectral efficiency, present user fairness and grant free access to sixth generation (6G) vehicular networks. This paper proposes a new optimization framework for NOMAenabled cooperative vehicular network. In particular, we jointly optimize the vehicle paring, channel assignment, and power allocation at source and relaying vehicles. The objective is to maximize the sum rate of the system subject to the power allocation, minimum rate, relay battery lifetime and successive interference cancellation constraints. To solve the joint optimization problem efficiently, we adopt dual theory followed by Karush-Kuhn-Tucker (KKT) conditions, where the dual variables are iteratively computed through sub-gradient method. Two less complex suboptimal optimization schemes are also presented as the benchmark cooperative vehicular schemes. Simulation results show the importance of the proposed joint optimization scheme compared to the other benchmark cooperative vehicular schemes.
\end{abstract}

Index Terms-Sixth generation (6G), Vehicular networks, Nonorthogonal multiple access (NOMA), Optimal resource allocation, Successive interference cancellation (SIC).

\section{INTRODUCTION}

The exponentially growing number of connected vehicles has brought an unconventional change in sixth generation (6G) intelligent transportation systems [1], [2]. The aim of advance transportation systems is to support massive vehicles connections and high transmission rate. Recently, 3rd generation partnership project (3GPP) is also working on public safety intelligent transportation solutions [3]. Although orthogonal multiple access (OMA) techniques [4] have served as successful multiplexing scheme in existing transportation networks, it seems less capable to meet the requirements of the large-scale vehicular networks [5]. Several solutions have been proposed ranging from exploring heterogeneous networks [6] to deploying multi antenna systems, among which non-orthogonal multiple access (NOMA) techniques have emerged as the attractive candidates and key enablers of advance vehicular networks [7]. It has been well-established

Z. Ali and G. A. S. Sidhu are with the Department of Electrical and Computer Engineering, COMSATS University Islamabad, Islamabad 44000, Pakistan. (emails: zainalihanan1@ gmail.com,guftaarahmad@comsats.edu.pk)

Wali Ullah Khan is with the Interdisciplinary Center for Security, Reliability and Trust (SnT)/ SigCom, University of Luxembourg, 4365 Esch-sur-Alzette, Luxembourg. (email: waliullah.khan@uni.lu)

A. Ihsan is with with Department of Information and Communication Engineering, Shanghai Jiao Tong University, Shanghai 200240, China. (email: ihsanasim@sjtu.edu.cn)

O. Waqar is with Thompson Rivers University (TRU), British Columbia (BC), Canada (email: owaqar@tru.ca).

Neeraj Kumar is with the Department of Computer Science and Engineering, Thapar Institute of Engineering, India. (email: neeraj.kumar@thapar.edu). that the NOMA techniques outperform the conventional OMA techniques in terms of spectral efficiency, quality of services (QoS) requirements and fairness [8], [9]. Existing NOMA techniques can be divided into two major categories, i.e., code division multiplexing [10] and power domain multiplexing [11]. The code division NOMA is based on distinguished spreading codes, whereas, multiple independent signals are superimposed and are transmitted on the same channel with different power levels for power domain NOMA [12]. Once received, the successive interference cancellation (SIC) is performed to decode the stronger signal first, thus forwarding the interference-free signal to the weak user [13]. Since power domain NOMA offers higher flexibility and less complexity compared to code domain NOMA techniques [14], it has attracted key focus in many recent works [15]-[18].

\section{A. Related Work}

Recently, various works have done on the performance analysis of NOMA-enabled vehicular networks. For example, the authors of [19] designed a system of cognitive radio with simultaneous wireless information and power transfer for NOMA-enabled vehicular networks. Their objective was to obtained the exact expression for outage probability of vehicles. In [20], the authors investigated the performance of two-user NOMA-enabled vehicular networks. They first derived the outage probability for the case when the system rate falls below the target rate and for the case when the rate of NOMA system drops than the OMA rate. Then, they also derived the expressions of average bit error rate and ergodic capacity. The work in [21] considered a full duplex communication in device-to-device aided vehicular networks. They proposed NOMA-enabled roadside units that operate as relay nodes. The authors derived the exact and closed-form expressions where the objective was to enhance the ergodic capacity of the system. The work of [22] and [23] proposed the decentralized algorithms in full duplex vehicular networks. They derived closed-form expressions for the exact capacity of the NOMA system in both urban and crowded vehicular scenarios.

Beside the above mentioned studies, several optimization problems have also investigated in NOMA-enabled vehicular networks. For instance, Khan et al. [24] integrated backscatter communication with multi-cell NOMA vehicular network to maximize the total achievable energy efficiency. To maximize the total capacity of device-to-device aided vehicular networks, the authors in [25] proposed a hyper-graph based solution. For cooperative vehicular systems the authors in [26] proposed a 


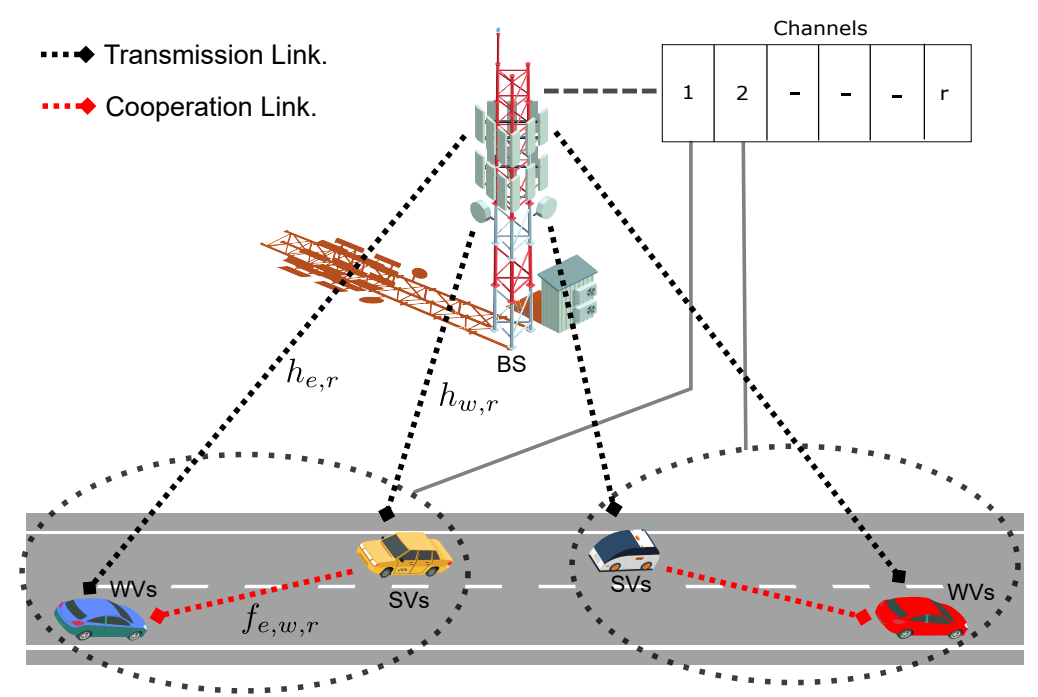

Fig. 1: System model of NOMA-enabled cooperative vehicular network.

resource allocation framework to maximize the sum rate of the system. In their considered system, an unmanned aerial vehicle serves as an amplify and forward relay. The authors in [27] optimized resource allocation to achieve fairness in NOMA-enabled vehicular networks. In [28], the authors proposed a sum rate maximization framework for NOMA-enabled vehicular communications. The work in [29] designed an efficient power control algorithm for NOMA-enabled vehicles. To improve the reliability and reduce the latency, the authors of [30] proposed an iterative distribution based algorithm. The work of [31] optimized spectrum allocation and power loading to enhance the reliability of NOMA-enabled vehicular networks. For NOMA vehicular communications, the authors in [32] studied the trade-off between energy efficiency and sum rate of the system. The authors in [33] worked on maximizing the energy efficiency of vehicular NOMA network. Moreover, the work in [34] studied the vehicular NOMA systems from the perspective of network security. Of late, Khan et al. [35] proposed an optimization framework for backscatter-aided vehicular networks to maximize the sum capacity while taken into account the minimum rate of each vehicle.

\section{B. Motivation and Contributions}

As discussed above, the problem of cooperative vehicular communication using power domain NOMA has not been studied well in the literature. In typical NOMA systems, the vehicle with better channel gain, i.e., the strong vehicle always decodes the signal of the weak vehicle, i.e., a vehicle with the lower value of channel gain, and perform the SIC operation. This also provides an opportunity for cooperative NOMA transmission as the strong vehicle always has the symbol of weak vehicle and can help in relaying. It is anticipated that this would also remove the additional cost of deploying independent relaying units. To the best of our knowledge, the problem that jointly optimizes the vehicle pairing, channel assignment, and power allocation at source and relaying vehicle to maximize the sum rate of has not yet been investigated. To fill this gap, we provide a new joint optimization framework to improve the performance of NOMA-enabled vehicular network. The efficient solutions are derived by dual theory followed by Karush-Kuhn-Tucker (KKT) conditions, where the dual variables are iteratively computed through sub-gradient method. Simulation results demonstrate the superiority of the proposed optimization scheme over the other benchmark schemes. The major contributions of this paper are given below:

- A new network setup is considered where a source serves multiple vehicles in downlink through multiple channels. Meanwhile, the strong vehicles also cooperate with weak vehicles by relaying their data using decode and forward (DF) protocols. We aim to jointly optimize the vehicle pairing, channel assignment, and power allocation at source and relaying vehicles.

- To maximize the sum-rate of the cooperative vehicular NOMA network, we formulate a joint optimization problem while keeping in account the rate requirement of each vehicle, battery capacity, and SIC constraints. We use dual theory followed by KKT conditions to obtain the efficient solutions.

- Two less complex solutions are also presented; 1) Vehicle paring, channel assignment, and relaying power are optimized under the equal power allocation at each vehicle pair; 2) Channel assignment, Power allocation at source and relaying vehicles for predefined vehicle pairing.

The remaining of the paper is organized as follows. In Section II the system model is explained. Section III presents the mathematical formulation and solution of the proposed. An intuition based solution is presented in Section IV. Discussion on simulation results is provided in Section V. Finally, the work is concluded in Section VI.

\section{System Model ANd Assumptions}

A downlink transmission of vehicular communication is assumed where a BS is serving $\Lambda$ vehicles on $\Lambda / 2$ channels, as shown in Fig. 1. We consider NOMA based vehicular 
communication where each channel is shared by two vehicles ${ }^{1}$. This work assume that: 1) We consider the perfect channel state information of vehicles at BS to focus on vehicle pairing, channel allocation, and power control at BS and near vehicles in this work [37]; 2) The channels consider in this network are undergone independent and identical Rayleigh fading [38]; 3) The BS and vehicles are equipped with a single antenna as considered in [39], [40]. The BS uses superposition coding to transmit the data to the vehicles. If the $w$ th and eth vehicles are sharing the $r$ th channel, then the received signals are given as

$$
\begin{aligned}
& t_{w}=h_{w, r}\left(\sqrt{p_{w, r}} s_{w}+\sqrt{p_{e, r}} s_{e}\right)+n_{w}, \\
& t_{e}=h_{e, r}\left(\sqrt{p_{e, r}} s_{e}+\sqrt{p_{w, r}} s_{w}\right)+n_{e},
\end{aligned}
$$

where $s_{w}, s_{e}, h_{w, r}$ and $h_{e, r}$ represent the transmitted symbols of the $w$ th and $e$ th vehicle, and the complex channel coefficients from the BS to the $w$ th and $e$ th vehicles at the $r$ th channel, respectively. The powers allocated for the transmission of the $w$ th and $e$ th vehicle sharing the $r$ th channel are denoted as $p_{w, r}$ and $p_{e, r}$, respectively. Moreover, $n_{w}$ and $n_{e}$ represent the additive white Gaussian noise (AWGN) at the respective vehicles.

In the proposed system the strong vehicles (SVs) cooperate with the weak vehicles (WVs) by relaying their data. The entire time slot is divided into two equal parts. In the first interval, the BS transmits the data to all the vehicles. In the second interval the SVs relay the data to the WVs after reencoding the information with a different set of codewords as compared to the BS. Thus, WVs can employ code combining and the sum rate of a WV can be written as the sum of mutual information in both time slots [41]- [43]. Hence, under this cooperative transmission the rate of the $w$ th vehicle is given as [44]

$$
\begin{aligned}
& R_{w}=\frac{1}{2} \log _{2}\left(1+\frac{p_{w, r}\left|h_{w, r}\right|^{2}}{\left(1-y_{w}\right) p_{e, r}\left|h_{w, r}\right|^{2}+\sigma^{2}}\right)+\left(1-y_{w}\right) \min \left(\frac{1}{2}\right. \\
& \left.\log _{2}\left(1+\frac{P r_{e, w, r}\left|f_{e, w, r}\right|^{2}}{\sigma^{2}}\right), \frac{1}{2} \log _{2}\left(1+\frac{p_{w, r}\left|h_{e, r}\right|^{2}}{p_{e, r}\left|h_{e, r}\right|^{2}+\sigma^{2}}\right)\right),
\end{aligned}
$$

where $y_{w}=1$ if $w$ belongs to the set of all strong vehicles, denoted as $\boldsymbol{S}$, and $y_{w}=0$ otherwise. Vehicle- $e$ and vehicle$w$ are considered to be sharing the same channel. The power allocated by the BS for the transmission of the $w$ th vehicle at the $r$ th channel is denoted by $p_{w, r}$. The power invested by the $w$ th SV to relay the data of the eth vehicle at the $r$ th channel is denoted as $\operatorname{Pr}_{e, w, r}$. While $\left|h_{w, r}\right|^{2}$ and $\left|f_{e, w, r}\right|^{2}$ are the gains of the $r$ th channel from the BS to the $w$ th vehicle and from the $w$ th to the $e$ th vehicle, respectively. The variance of AWGN is denoted by $\sigma^{2}$.

We aim to optimize vehicle pairing, channel assignment, and power allocation such that the overall rate of the system can be maximized. The available spectrum is divided into $\Lambda / 2$ channels of equal bandwidth. The objective function can be

\footnotetext{
${ }^{1}$ Hardware complexity and processing delay due to SIC increases with the increasing number of vehicles per channel. Thus, the proposed system model consists of two vehicles per channel [36].
}

written mathematically as

$$
\max _{p_{w, r}, x_{w, e, r}, y_{e}, P r_{e, w, r}} \sum_{w=1}^{\Lambda} \sum_{e=1}^{\Lambda} \sum_{r=1}^{\Lambda / 2} \frac{2 B x_{w, e, r}}{\Lambda} R_{w},
$$

where $B$ is the total available bandwidth. Each channel is shared by two vehicles and there are $\Lambda$ number of vehicles in the system. Thus, the bandwidth of every channel is given by $\frac{2 B}{\Lambda}$. The binary variable $x_{w, e, r}$ in (1) facilitates the mathematical formulation and is defined as

$$
x_{w, e, r}= \begin{cases}1, & \begin{array}{l}
\text { if the } w \text { th user is paired with the } e \text { th user at } \\
\text { the } r \text { th channel, }
\end{array} \\
0, & \text { otherwise. }\end{cases}
$$

It is required that each vehicle should pair with one and only one other vehicle, which is guaranteed by

$$
\sum_{e=1}^{\Lambda} \sum_{r=1}^{\Lambda / 2} x_{w, e, r}=1, \forall w
$$

The need of only one vehicle pair per channel dictates

$$
\sum_{w=1}^{\Lambda} \sum_{e=1}^{\Lambda} x_{w, e, r}=2, \forall r
$$

To ensure that each pair has one SV and only one WV, we have

$$
\sum_{w=1}^{\Lambda} \sum_{e=1}^{\Lambda} y_{w} x_{w, e, r}=1, \forall r
$$

We assume that exactly half of the vehicles in the system are labeled as SVs, which is guaranteed by

$$
\sum_{w=1}^{\Lambda} y_{w}=\frac{\Lambda}{2}
$$

The BS and the SVs have limited power budgets. Thus, we have

$$
\begin{aligned}
& \sum_{w=1}^{\Lambda} \sum_{e=1}^{\Lambda} \sum_{r=1}^{\Lambda / 2} x_{w, e, r} p_{w, r} \leq P_{T} \\
& \sum_{e=1}^{\Lambda} \sum_{r=1}^{\Lambda / 2} y_{w} x_{w, e, r} P r_{e, w, r} \leq P \max _{w}, \forall w
\end{aligned}
$$

where the maximum allowed transmit powers at the BS and the $w$ th $\mathrm{SV}$ are represented as $P_{T}$ and $P \max _{w}$, respectively. For successful SIC, it is required that the received power of the WV's signal should be greater than that of the SV's signal. Mathematically, it is given as

$$
\sum_{e=1}^{\Lambda} \sum_{r=1}^{\Lambda / 2} x_{w, e, r} y_{w}\left(p_{e, r}-p_{w, r}\right) \geq \psi_{w, r}, \forall w,
$$

where $\psi_{w, r}=\frac{\bar{Y}}{\left|h_{w, r}\right|^{2}}$, and $\bar{Y}$ is the minimum required gap in the received powers at the $\mathrm{SV}$. Let $R t_{w}$ be the minimum acceptable rate of the $w$ th vehicle. Then, for the feasibility of 
the solution, we must guarantee

$$
\sum_{e=1}^{\Lambda} \sum_{r=1}^{\Lambda / 2} \frac{2 B x_{w, e, r}}{\Lambda} R_{w} \geq R t_{w}, \forall w,
$$

where $R t_{w}$ is the minimum acceptable data rate of the $w$ th vehicle.

\section{Problem Formulation And Proposed Solution}

The achievable rate in the case of DF relaying is the minimum of the rates on both links. Under the proposed cooperative DF transmission, the rate at the second hop should not exceed the received rate at the first hop. Thus, for each $\mathrm{SV}$ the relaying constraint is written as

$$
\begin{aligned}
& \frac{B x_{w, e, r} y_{w}}{\Lambda}\left(\log _{2}\left(1+\frac{p_{e, r}\left|h_{w, r}\right|^{2}}{p_{w, r}\left|h_{w, r}\right|^{2}+\sigma^{2}}\right) \geq\right. \\
& \left.\log _{2}\left(1+\frac{P r_{e, w, r}\left|f_{e, w, r}\right|^{2}}{\sigma^{2}}\right)\right), \forall w, e, r .
\end{aligned}
$$

With this, the problem of optimizing resource allocation to maximize the sum-rate is given by

$$
\begin{aligned}
& \text { P1: } \max _{p_{w, r}, x_{w, e, r}, y_{e}, P r_{e, w, r}} \sum_{w=1}^{\Lambda} \sum_{e=1}^{\Lambda} \sum_{r=1}^{\Lambda / 2} \frac{2 B x_{w, e, r}}{\Lambda} \\
& \left(\frac{1}{2} \log _{2}\left(1+\frac{p_{w, r}\left|h_{w, r}\right|^{2}}{y_{e} p_{e, r}\left|h_{w, r}\right|^{2}+\sigma^{2}}\right)\right. \\
& \left.+y_{e} \frac{1}{2} \log _{2}\left(1+\frac{P r_{e, w, r}\left|f_{e, w, r}\right|^{2}}{\sigma^{2}}\right)\right) \text {, } \\
& \text { s.t. } \\
& \sum_{e=1}^{\Lambda} \sum_{r=1}^{\Lambda / 2} \frac{2 B x_{w, e, r}}{\Lambda}\left(\frac{1}{2} \log _{2}\left(1+\frac{p_{w, r}\left|h_{w, r}\right|^{2}}{y_{e} p_{e, r}\left|h_{w, r}\right|^{2}+\sigma^{2}}\right)\right. \\
& \left.+y_{e} \frac{1}{2} \log _{2}\left(1+\frac{P r_{e, w, r}\left|f_{e, w, r}\right|^{2}}{\sigma^{2}}\right)\right) \geq R t_{w},
\end{aligned}
$$

and $(2)-(10)$.

This problem is a mixed binary integer programming and has non-trivial solution. To make P1 more tractable, we reformulate the problem such that the power allocation at the BS is obtained in two steps. Firstly, the power allocation for each vehicle pair is calculated. Secondly, the shares of transmission powers of SV and WV are obtained. Without loss of generality, we assume that all the vehicles are sorted in descending order of their channel gains. Thus, replacing $\sum_{e=1}^{\Lambda}$ with $\sum_{e=w+1}^{\Lambda}$ ensures that in expression $\sum_{w=1}^{\Lambda} \sum_{e=w+1}^{\Lambda} \sum_{r=1}^{\Lambda / 2} x_{w, e, r}$, the subscript $w$ will always represent an SV and $e$ will denote the $\mathrm{WV}$ in the pair. This eliminates the requirement of binary variable $y_{w}$. The power allotted to the $(n, m)$ th vehicle pair at the $r$ th channel is denoted as $P_{w, e, r}$. The fractions of $P_{w, e, r}$ allocated for the transmission of $\mathrm{SV}$ and $\mathrm{WV}$ are denoted as $\eta_{w, e, r}$ and $\left(1-\eta_{w, e, r}\right)$, respectively. Then, the problem is written as

$$
\text { P2: } \begin{aligned}
\max _{P_{w, e, r}, P r_{e, w, r}, \eta_{w, e, r}, x_{w, e, r}} & \sum_{w=1}^{\Lambda} \sum_{e=w+1}^{\Lambda} \sum_{r=1}^{\Lambda / 2} \frac{B}{\Lambda / 2} \frac{x_{w, e, r}}{2} \\
& \left(\log _{2}\left(1+\frac{\eta_{w, e, r} P_{w, e, r}\left|h_{w, r}\right|^{2}}{\sigma^{2}}\right)\right. \\
& +\log _{2}\left(\frac{P r_{e, w, r}\left|f_{e, w, r}\right|^{2}}{\sigma^{2}}+1\right) \\
& \left.+\log _{2}\left(1+\frac{\left(1-\eta_{w, e, r}\right) P_{w, e, r}\left|h_{e, r}\right|^{2}}{\eta_{w, e, r} P_{w, e, r}\left|h_{e, r}\right|^{2}+\sigma^{2}}\right)\right),
\end{aligned}
$$

s.t.

$\sum_{w=1}^{\Lambda} \sum_{e=w+1}^{\Lambda} \sum_{r=1}^{\Lambda / 2} x_{w, e, r} P_{w, e, r} \leq P_{T}$,

$\sum_{e=w+1}^{\Lambda} \sum_{r=1}^{\Lambda / 2} x_{w, e, r} \operatorname{Pr}_{e, w, r} \leq \operatorname{Pmax}_{w}, \forall w$,

$$
\begin{aligned}
& \sum_{w=1}^{\Lambda} \sum_{e=w+1}^{\Lambda} x_{w, e, r}\left(\left(1-\eta_{w, e, r}\right) P_{w, e, r}-\eta_{w, e, r} P_{w, e, r}\right) \\
& \geq \psi_{w, r}, \forall r,
\end{aligned}
$$

$$
\begin{aligned}
& \sum_{e=w+1}^{\Lambda} \sum_{r=1}^{\Lambda / 2} \frac{B x_{w, e, r}}{\Lambda} \log _{2}\left(1+\frac{\eta_{w, e, r} P_{w, e, r}\left|h_{w, r}\right|^{2}}{\sigma^{2}}\right) \\
& \geq R t_{w}, \forall w \in S,
\end{aligned}
$$

$$
\begin{aligned}
& \sum_{w=1}^{\Lambda} \sum_{r=1}^{\Lambda / 2} \frac{B x_{w, e, r}}{\Lambda}\left(\log _{2}\left(1+\frac{\left(1-\eta_{w, e, r}\right) P_{w, e, r}\left|h_{e, r}\right|^{2}}{\eta_{w, e, r} P_{w, e, r}\left|h_{e, r}\right|^{2}+\sigma^{2}}\right)\right. \\
& \left.+\log _{2}\left(1+\frac{P r_{e, w, r}\left|f_{e, w, r}\right|^{2}}{\sigma^{2}}\right)\right) \geq R t_{w}, \forall e>w, e \in W,
\end{aligned}
$$

$$
\begin{aligned}
& \frac{B x_{w, e, r}}{\Lambda}\left(\log _{2}\left(1+\frac{\left(1-\eta_{w, e, r}\right) P_{w, e, r}\left|h_{w, r}\right|^{2}}{\eta_{w, e, r} P_{w, e, r}\left|h_{w, r}\right|^{2}+\sigma^{2}}\right) \geq\right. \\
& \left.\log _{2}\left(1+\frac{P r_{e, w, r}\left|f_{e, w, r}\right|^{2}}{\sigma^{2}}\right)\right), \forall w, r, e>w
\end{aligned}
$$$$
\sum_{e=w+1}^{\Lambda} \sum_{r=1}^{\Lambda / 2} x_{w, e, r}=1, \forall w \in S,
$$$$
\sum_{w=1}^{\Lambda} \sum_{e=w+1}^{\Lambda} x_{w, e, r}=1, \forall r
$$

$$
\sum_{w=1}^{\Lambda} \sum_{r=1}^{\Lambda / 2} x_{w, e, r}=1, \forall e>w, e \in W,
$$

where $W$ represents the set of WVs. Note that the transfor- 


$$
\begin{aligned}
& L\left(P_{w, e, r}, \operatorname{Pr}_{e, w, r}, \eta_{w, e, r}, x_{w, e, r}, \mu_{w}, \bar{\mu}_{e}, \epsilon_{w, e, r}, \tau_{r}, \lambda, \bar{\lambda}_{w}\right)=\sum_{w=1}^{\Lambda} \sum_{e=w+1}^{\Lambda} \sum_{r=1}^{\Lambda / 2} \frac{B x_{w, e, r}}{\Lambda}\left[\log _{2}\left(1+\frac{\eta_{w, e, r} P_{w, e, r}\left|h_{w, r}\right|^{2}}{\sigma^{2}}\right)\right. \\
& \left.+\log _{2}\left(1+\frac{\left(1-\eta_{w, e, r}\right) P_{w, e, r}\left|h_{e, r}\right|^{2}}{\eta_{w, e, r} P_{w, e, r}\left|h_{e, r}\right|^{2}+\sigma^{2}}\right)+\log _{2}\left(1+\frac{P r_{e, w, r}\left|f_{e, w, r}\right|^{2}}{\sigma^{2}}\right)\right]+\sum_{w=1}^{\Lambda} \mu_{w}\left[\sum_{e=w+1}^{\Lambda} \sum_{r=1}^{\Lambda / 2} \frac{B x_{w, e, r}}{\Lambda} \log _{2}\right. \\
& \left.\left(1+\frac{\eta_{w, e, r} P_{w, e, r}\left|h_{w, r}\right|^{2}}{\sigma^{2}}\right)-R t_{w}\right]+\sum_{e=w+1}^{\Lambda} \bar{\mu}_{e}\left[\sum _ { w = 1 } ^ { \Lambda } \sum _ { r = 1 } ^ { \Lambda / 2 } \frac { B x _ { w , e , r } } { \Lambda } \left(\log _{2}\left(1+\frac{\left(1-\eta_{w, e, r}\right) P_{w, e, r}\left|h_{e, r}\right|^{2}}{\eta_{w, e, r} P_{w, e, r}\left|h_{e, r}\right|^{2}+\sigma^{2}}\right)\right.\right. \\
& \left.\left.+\log _{2}\left(1+\frac{P r_{e, w, r}\left|f_{e, w, r}\right|^{2}}{\sigma^{2}}\right)\right)-R t_{w}\right]+\sum_{w=1}^{\Lambda} \sum_{e=w+1}^{\Lambda} \sum_{r=1}^{\Lambda / 2} \frac{\epsilon_{w, e, r} B x_{w, e, r}}{\Lambda}\left[\log _{2}\left(1+\frac{\left(1-\eta_{w, e, r}\right) P_{w, e, r}\left|h_{w, r}\right|^{2}}{\eta_{w, e, r} P_{w, e, r}\left|h_{w, r}\right|^{2}+\sigma^{2}}\right)\right. \\
& \left.-\log _{2}\left(\frac{\operatorname{Pr}_{e, w, r}\left|f_{e, w, r}\right|^{2}}{\sigma^{2}}+1\right)\right]+\sum_{r=1}^{\Lambda / 2} \tau_{r}\left[\sum_{w=1}^{\Lambda} \sum_{e=w+1}^{\Lambda} x_{w, e, r}\left(P_{w, e, r}-2 \eta_{w, e, r} P_{w, e, r}\right)-\psi_{w, r}\right] \\
& +\lambda\left[P_{T}-\sum_{w=1}^{\Lambda} \sum_{e=w+1}^{\Lambda} \sum_{r=1}^{\Lambda / 2} x_{w, e, r} P_{w, e, r}\right]+\sum_{w=1}^{\Lambda} \bar{\lambda}_{w}\left[\operatorname{Pmax}_{w}-\sum_{e=w+1}^{\Lambda} \sum_{r=1}^{\Lambda / 2} x_{w, e, r} \operatorname{Pr}_{e, w, r}\right] .
\end{aligned}
$$

$$
\begin{aligned}
& \theta_{w, e, r}\left(P_{w, e, r}, \eta_{w, e, r}, P r_{e, w, r}\right)=\frac{B}{\Lambda}\left[\log _{2}\left(1+\frac{\eta_{w, e, r} P_{w, e, r}\left|h_{w, r}\right|^{2}}{\sigma^{2}}\right)+\log _{2}\left(1+\frac{\left(1-\eta_{w, e, r}\right) P_{w, e, r}\left|h_{e, r}\right|^{2}}{\eta_{w, e, r} P_{w, e, r}\left|h_{e, r}\right|^{2}+\sigma^{2}}\right)\right. \\
& \left.+\log _{2}\left(1+\frac{P r_{e, w, r}\left|f_{e, w, r}\right|^{2}}{\sigma^{2}}\right)\right]+\mu_{w} \frac{B}{\Lambda} \log _{2}\left(1+\frac{\eta_{w, e, r} P_{w, e, r}\left|h_{w, r}\right|^{2}}{\sigma^{2}}\right) \bar{\mu}_{e} \frac{B}{\Lambda}\left[\log _{2}\left(1+\frac{\left(1-\eta_{w, e, r}\right) P_{w, e, r}\left|h_{e, r}\right|^{2}}{\eta_{w, e, r} P_{w, e, r}\left|h_{e, r}\right|^{2}+\sigma^{2}}\right)\right. \\
& \left.+\log _{2}\left(\frac{P r_{e, w, r}\left|f_{e, w, r}\right|^{2}}{\sigma^{2}}+1\right)\right]+\epsilon_{w, e, r} \frac{B}{\Lambda}\left[\log _{2}\left(1+\frac{\left(1-\eta_{w, e, r}\right) P_{w, e, r}\left|h_{w, r}\right|^{2}}{\eta_{w, e, r} P_{w, e, r}\left|h_{w, r}\right|^{2}+\sigma^{2}}\right)-\log _{2}\left(1+\frac{P r_{e, w, r}\left|f_{e, w, r}\right|^{2}}{\sigma^{2}}\right)\right] \\
& +\tau_{r}\left(P_{w, e, r}-2 \eta_{w, e, r} P_{w, e, r}\right)-\lambda P_{w, e, r}-\bar{\lambda}_{w} \operatorname{Pr}_{e, w, r} .
\end{aligned}
$$

mation to P2 has made the problem more tractable. However the problem is still non-convex ${ }^{2}$ and we then apply the duality theory to find the efficient solution ${ }^{3}$ in the case of mix integer programming problems. The corresponding dual problem is given as

$$
\begin{aligned}
& \min _{\mu_{w}, \bar{\mu}_{e}, \epsilon_{w, e, r}, \tau_{r}, \lambda, \bar{\lambda}_{w}} D\left(\mu_{w}, \bar{\mu}_{e}, \epsilon_{w, e, r}, \tau_{r}, \lambda, \bar{\lambda}_{w}\right) \\
& \text { s.t. } \quad \mu_{w} \geq 0, \bar{\mu}_{e} \geq 0, \epsilon_{w, e, r} \geq 0, \tau_{r} \geq 0, \lambda \geq 0, \bar{\lambda}_{w} \geq 0,
\end{aligned}
$$

where $\mu_{w}, \bar{\mu}_{e}, \epsilon_{w, e, r}, \tau_{r}, \lambda, \bar{\lambda}_{w}$ are the dual variables. The dual function is defined as

$$
\begin{aligned}
& D\left(\mu_{w}, \bar{\mu}_{e}, \epsilon_{w, e, r}, \tau_{r}, \lambda, \bar{\lambda}_{w}\right)=\max _{P_{w, e, r}, P r_{e, w, r}, \eta_{w, e, r}} L\left(P_{w, e, r},\right. \\
& \left.\operatorname{Pr}_{e, w, r}, \eta_{w, e, r}, x_{w, e, r}, \mu_{w}, \bar{\mu}_{e}, \epsilon_{w, e, r}, \tau_{r}, \lambda, \bar{\lambda}_{w}\right), \\
& \text { s.t.(20),(21),(22), }
\end{aligned}
$$

where $L$ is called the Lagrangian and is given in (25) on the

\footnotetext{
${ }^{2} \mathrm{~A}$ discussion on the convexity of the problem is provided in the appendix. ${ }^{3}$ Duality theory based solution guarantees convergence to a local maxima [45].
}

top of previous page. Rewriting the Lagrangian, we get

$$
\begin{aligned}
& L=\sum_{w=1}^{\Lambda} \sum_{e=w+1}^{\Lambda} \sum_{r=1}^{\Lambda / 2} x_{w, e, r} \theta_{w, e, r}\left(P_{w, e, r}, \eta_{w, e, r}, P_{w, e, r}\right)-\sum_{w=1}^{\Lambda} \\
& \mu_{w} R t_{w}-\sum_{e=w+1}^{\Lambda} \mu_{e} R t_{w}-\sum_{r=1}^{\Lambda / 2} \tau_{r} \psi_{w, r}+\lambda P_{T}+\sum_{w=1}^{\Lambda} \bar{\lambda}_{w} \operatorname{Pmax}_{w}
\end{aligned}
$$

where $\theta_{w, e, r}\left(P_{w, e, r}, \eta_{w, e, r}, \operatorname{Pr}_{e, w, r}\right)$ is defined as (27) on the top of this page.

For a given value of $x_{w, e, r}$, (24) can be decomposed into following $\left(\left(\sum_{1=0}^{\Lambda-1} \Lambda-i\right) \frac{\Lambda}{2}\right)$ sub-problems:

$D\left(\mu_{w}, \bar{\mu}_{e}, \epsilon_{w, e, r}, \tau_{r}, \lambda, \bar{\lambda}_{w}\right)=$

$\max _{P_{w, e, r}, P r_{e, w, r}, \eta_{w, e, r}} \theta_{w, e, r}\left(P_{w, e, r}, \eta_{w, e, r}, P r_{e, w, r}\right), \forall w, k, m>n$.

Applying Karush Kuhn Tucker (KKT) conditions [46] to (28), we get (29) on the top of the page, where $\beta_{w}=$ $\frac{B+\mu_{w} B}{\Lambda}, \phi_{e}=\frac{B+\bar{\mu}_{e} B}{\Lambda}, \rho_{w, e, r}=\frac{\epsilon_{w, e, r} B}{\Lambda}$ and $\Theta_{r}=$ $\tau_{r}\left(1-2 \eta_{w, e, r}\right)-\lambda$. We obtain the solution of $P_{w, e, r}^{*}$, by selecting the maximum root that satisfies (29). By differentiating 


$$
\begin{aligned}
& P_{w, e, r}^{4}\left(\eta_{w, e, r}^{2} h_{e, r}^{2} h_{w, r}^{2} \Theta_{r}\right)+P_{w, e, r}^{3}\left(\eta_{w, e, r} h_{e, r} h_{w, r}\left(\left(h_{e, r}+h_{w, r}\right) \Theta_{r} \sigma^{2}+\eta_{w, e, r}\left(\beta_{w} h_{e, r} h_{w, r}+\left(h_{e, r}+h_{w, r}\right) \Theta_{r} \sigma^{2}\right)\right)\right) \\
& +P_{w, e, r}^{2}\left(\sigma ^ { 2 } \left(h_{e, r} h_{w, r} \Theta_{r} \sigma^{2}+\eta_{w, e, r}^{2} h_{e, r} h_{w, r}\left(-\rho_{w, e, r} h_{e, r}-\phi_{e} h_{w, r}+\beta_{w}\left(h_{e, r}+h_{w, r}\right)+\Theta_{r} \sigma^{2}\right)+\eta_{w, e, r}\left(\rho_{w, e, r} h_{e, r}^{2}\right.\right.\right. \\
& \left.\left.\left.h_{w, r}+\beta_{w} h_{e, r} h_{w, r}^{2}+\phi_{e} h_{e, r} h_{w, r}^{2}+h_{e, r}^{2} \Theta_{r} \sigma^{2}+2 h_{e, r} h_{w, r} \Theta_{r} \sigma^{2}+h_{w, r}^{2} \Theta_{r} \sigma^{2}\right)\right)\right)+P_{w, e, r}\left(\sigma ^ { 4 } \left(c h_{e, r} h_{w, r}+\eta_{w, e, r}^{2}\left(\beta_{w}\right.\right.\right. \\
& \left.\left.\left.-\phi_{e}-\rho_{w, e, r}\right) h_{e, r} h_{w, r}+\rho_{w, e, r} h_{e, r} h_{w, r}+h_{e, r} \Theta_{r} \sigma^{2}+h_{w, r} \Theta_{r} \sigma^{2}+\eta_{w, e, r}\left(h_{e, r}+h_{w, r}\right)\left(\beta_{w} h_{w, r}+\Theta_{r} \sigma^{2}\right)\right)\right) \\
& +\sigma^{6}\left(\phi_{e}\left(h_{e, r}-\eta_{w, e, r} h_{e, r}\right)+\eta_{w, e, r}\left(\beta_{w}-\rho_{w, e, r}\right) h_{w, r}+\rho_{w, e, r} h_{w, r}+\Theta_{r} \sigma^{2}\right)=0 .
\end{aligned}
$$

(28) with respect to $\eta_{w, e, r}$ we get the following solution:

$$
\eta_{w, e, r}^{*}=\left(\frac{-\omega+\sqrt{\omega^{2}-4 \Gamma \Delta}}{2 \Gamma}\right)^{+}
$$

where $A^{+}=\max (0, A)$ and

$$
\begin{gathered}
\Gamma=h_{e, r} h_{w, r} P_{w, e, r}^{2}\left(h_{e, r} P_{w, e, r}+\sigma^{2}\right)\left(h_{w, r} P_{w, e, r}+\sigma^{2}\right) \Omega_{w, e, r}, \\
\Delta=\sigma^{2}\left(h_{e, r} P_{w, e, r}+\sigma^{2}\right)\left(h_{w, r} P_{w, e, r}+\sigma^{2}\right)\left(h_{e, r} P_{w, e, r} V_{e}+\right. \\
\left.h_{w, r} P_{w, e, r}\left(U_{w}+W_{w, e, r}\right)+\sigma^{2} \Omega_{w, e, r}\right), \\
\omega=P_{w, e, r}\left(h_{e, r} P_{w, e, r}+\sigma^{2}\right)\left(h_{w, r} P_{w, e, r}+\sigma^{2}\right)\left(h_{w, r} \sigma^{2}\right. \\
\left.\Omega_{w, e, r}+h_{e, r}\left(h_{w, r} P_{w, e, r}\left(U_{w}+V_{e}+W_{w, e, r}\right)+\sigma^{2} \Omega_{w, e, r}\right)\right)
\end{gathered}
$$

and $\Omega_{w, e, r}=-2 \tau_{w, e, r} P_{w, e, r}, U_{e}=\frac{B+\mu_{w} B}{\Lambda}, V_{e}=$ $\frac{-B-\bar{\mu}_{e} B}{\Lambda}, W_{w, e, r}=\frac{-\epsilon_{w, e, r} B}{\Lambda}$.

To find the solution of power allocation for relaying at each SV, we differentiate (27) with respect to $P r_{e, w, r}$ and obtain:

$$
\operatorname{Pr}_{e, w, r}^{*}=\left(\frac{\frac{B-\epsilon_{w, e, r} B}{\Lambda}+\bar{\mu}_{e}}{\bar{\lambda}_{w}}-\frac{\sigma^{2}}{f_{e, w, r}}\right)^{+} .
$$

Now for the solution of $x_{w, e, r}$ we substitute $P_{w, e, r}^{*}, \eta_{w, e, r}^{*}$ and $\operatorname{Pr}_{e, w, r}^{*}$ in (24):

$D=\max _{x_{w, e, r}}\left\{\sum_{w=1}^{\Lambda} \sum_{e=w+1}^{\Lambda} \sum_{r=1}^{\Lambda / 2} \theta_{w, e, r}\left(P_{w, e, r}^{*}, \eta_{w, e, r}^{*}, P r_{e, w, r}^{*}\right) x_{w, e, r}\right.$

$$
-\sum_{w=1}^{\Lambda} \mu_{w} R t_{w}-\sum_{e=w+1}^{\Lambda} \mu_{e} R t_{w}-\sum_{r=1}^{\Lambda / 2} \tau_{r} \psi_{w, r}+\lambda P_{T}+\sum_{w=1}^{\Lambda} \bar{\lambda}_{w}
$$

$\operatorname{Pmax}_{w} \mid \sum_{e=w+1}^{\Lambda} \sum_{r=1}^{\Lambda / 2} x_{w, e, r}=1, \forall w, \sum_{w=1}^{\Lambda} \sum_{e=w+1}^{\Lambda} x_{w, e, r}=1, \forall r$, $\left.\sum_{w=1}^{\Lambda} \sum_{r=1}^{\Lambda / 2} x_{w, e, r}=1, \forall e>w\right\}$.

The solution of (32) is obtained by finding $w^{*}, e^{*}, r^{*}$ such that $\theta_{n^{*}, m^{*}, k^{*}}\left(P_{n^{*}, m^{*}, k^{*}}^{*}, \eta_{n^{*}, m^{*}, k^{*}}\right.$,

$\left.P r_{m^{*}, n^{*}, k^{*}}\right) \geq \theta_{w, e, r}\left(P_{w, e, r}^{*}, \eta_{w, e, r}^{*}, P r_{e, w, r}^{*}\right) \forall w, e, r$. This gives us the best user pair present in the system and the best channel for that pair. After this, we pair vehicle $n^{*}$ with $m^{*}$ and assign channel $k^{*}$ to the pair by setting $x_{n^{*}, m^{*}, k^{*}}=1$. Then we remove these vehicle and channel from the pairing and assignment process, and then find the next best pair and channel allocation. This process is repeated $\frac{\Lambda}{2}$ times. In vehicular networks the pairing and channel allocation may need to be changed when a vehicle changes direction, velocity or the distance from another vehicle. Thus, the pairing and channel assignment needs to be updated after every few iterations depending on the environment. In the case of high speed vehicles the pairing and assignment needs to be updated more often compared to slow speed vehicles. The benefit of using the proposed pairing and assignment scheme is that the performance improves in each iteration. If, instead a bruteforcing approach is used the performance of the system may be very poor until the algorithm has checked for all possible combination. Thus, in such cases it is advantageous to use to proposed scheme or the sub-optimal framework proposed in the later section.

Now it remains to solve the dual problem. We adopt subgradient method [47] to find the solution, where the gradients are given as:

$$
\begin{aligned}
\Delta_{\lambda}=\left(P_{T}-\sum_{w=1}^{\Lambda} \sum_{e=w+1}^{\Lambda} \sum_{r=1}^{\Lambda / 2} x_{w, e, r} P_{w, e, r}\right) \\
\Delta_{\bar{\lambda}_{w}}=\left(\operatorname{Pmax}_{w}-\sum_{e=w+1}^{\Lambda} \sum_{r=1}^{\Lambda / 2} x_{w, e, r} P r_{e, w, r}\right), \forall w, \\
\Delta_{\tau_{r}}=\left(\sum _ { w = 1 } ^ { \Lambda } \sum _ { e = w + 1 } ^ { \Lambda } x _ { w , e , r } \left(\left(1-\eta_{w, e, r}\right) P_{w, e, r}-\eta_{w, e, r}\right.\right. \\
\left.\left.P_{w, e, r}\right)-\psi_{w, r}\right) \forall r
\end{aligned}
$$




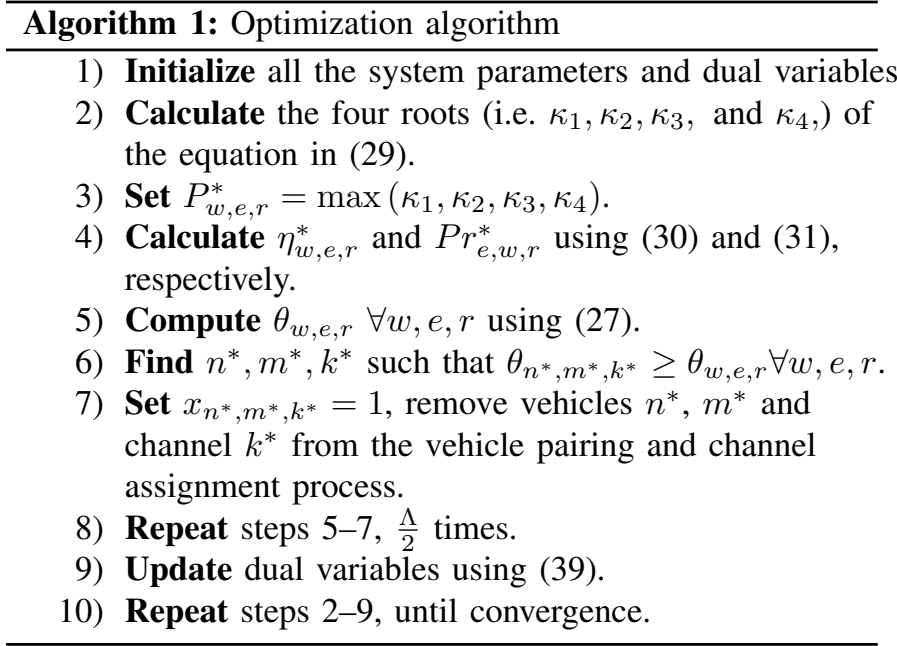

$$
\begin{gathered}
\Delta_{\mu_{w}}=\left(\sum_{e=w+1}^{\Lambda} \sum_{r=1}^{\Lambda / 2} \log _{2}\left(1+\frac{\eta_{w, e, r} P_{w, e, r}\left|h_{w, r}\right|^{2}}{\sigma^{2}}\right)\right. \\
\Delta_{\bar{\mu}_{e}}=\left(\sum_{w=1, r}^{\Lambda}-R t_{w}\right), \forall w \\
+\sum_{r=1}^{\Lambda / 2}\left(\log _{2}\left(1+\frac{\left(1-\eta_{w, e, r}\right) P_{w, e, r}\left|h_{e, r}\right|^{2}}{\eta_{w, e, r} P_{w, e, r}\left|h_{e, r}\right|^{2} \sigma^{2}}\right)\right. \\
\left.\left.\left.\left(1+\frac{P r_{e, w, r}\left|f_{e, w, r}\right|^{2}}{\sigma^{2}}\right)\right) \frac{B x_{w, e, r}}{\Lambda}-R t_{w}\right)\right), \forall e \\
\Delta_{\epsilon_{w, e, r}}=\left(\left(\log _{2}\left(1+\frac{\left(1-\eta_{w, e, r}\right) P_{w, e, r}\left|h_{w, r}\right|^{2}}{\eta_{w, e, r} P_{w, e, r}\left|h_{w, r}\right|^{2} \sigma^{2}}\right)-\right.\right. \\
\left.\left.\log _{2}\left(1+\frac{P r_{e, w, r}\left|f_{e, w, r}\right|^{2}}{\sigma^{2}}\right)\right) \frac{B x_{w, e, r}}{\Lambda}\right), \forall w, m, k
\end{gathered}
$$

The dual variable update at the $(i+1)$ th iteration is given as:

$$
\chi^{i+1}=\chi^{i}+\delta \Delta_{\chi},
$$

where $\delta$ is the step size and $\chi \in\left\{\lambda, \bar{\lambda}_{w}, \tau_{r}, \mu_{w}, \bar{\mu}_{e}, \epsilon_{w, e, r}\right\}$. The steps involved in finding the solution are summarized in the algorithm 1:

\section{LOW COMPLEXITY SOLUTION}

The framework proposed in section III provides the solution at the cost of high computational complexity. In this section we will design two less-complex optimization methods.

\section{A. Sub-Opt-1}

In the considered problem there are three types of power allocations: power allocated to each vehicle pair $\left(P_{w, e, r}\right)$, power distribution among the vehicle in each pair $\left(\eta_{w, e, r}\right)$ and power allocated by the SVs for relaying $\left(\operatorname{Pr}_{e, w, r}\right)$. The total computational complexity of finding these three power allocations is $O\left(\frac{3 \Lambda^{3}}{2}\right)$. As we are also optimizing the user pairing and channel allocation. Thus, if we allocate equal power to each user pair the optimization framework should be able to achieve promising results by optimizing $x_{w, e, r}, \alpha_{w, e, r}$ and $P r_{e, w, r}$ for the given value of $P_{w, e, r}$. In this case, the value of power allocated to each vehicle pair is given by: $P_{w, e, r}=\frac{2 P_{T}}{\Lambda}, \forall w, e, r$. Note that, fixing the values of $\eta_{w, e, r}$ and $P r_{e, w, r}$ may result in violating constraints (16) and (19), respectively. Hence, in this technique, we optimized these parameters in the same way as in section . The computational complexity of power allocation using the Equal Power framework is $O\left(\Lambda^{3}\right)$. As $O\left(\Lambda^{3}\right)<O\left(\frac{3 \Lambda^{3}}{2}\right)$, This method takes less time to converge. Further, for the fixed value of $P_{w, e, r}$, the problem of resource allocation becomes convex in nature. Thus, the optimal solution is guaranteed.

\section{B. Sub-Opt-2}

The proposed vehicle pairing and channel allocation scheme in Section III first requires to check the pairing of each vehicle with every other vehicle. This step has complexity of $O\left(\Lambda^{2}\right)$. Then we check the rate of each possible vehicle pair for every channel in the system. With $\Lambda / 2$ channels, the resultant complexity becomes $O\left(\frac{\Lambda^{3}}{2}\right)$. In this section, we propose a less complex algorithm, where vehicle pairing is obtained from intuitive method, then, Hungarian algorithm is applied for channel assignment. It is clear from intuition that, to achieve a specific value of rate, the faraway vehicle requires higher share of transmit power while the nearest vehicle could achieve the required rate in a comparatively small amount of power. Thus, to achieve overall better performance, the vehicle with strongest channel gain is paired to the vehicle with weakest channel [49]. Otherwise, if we pair together two vehicle with comparatively bad channel conditions, this specific pair would require a large amount of power to meet the rate requirement, leaving behind a very small fraction of power for the transmission of the vehicle with better channel conditions. So, we propose to pair the vehicle with best channel conditions (from the BS) with the vehicle having lowest value of channel gain, the second closest vehicle to the $\mathrm{BS}$ is paired to the vehicle with the second lowest value of the channel gain, and so on. Let the $i$ th SV be paired with the $j$ th $\mathrm{WV}$ and we denote this vehicle pair with variable $l$. Then, the sum rate of the $l^{*}$ th vehicle pair at the $r$ th channel can be written as:

$$
\begin{aligned}
& G\left(l^{*}, r\right)=\sum_{i=1}^{\Lambda} \sum_{j=i+1}^{\Lambda} \sum_{r=1}^{\Lambda / 2} \frac{B}{\Lambda / 2} \frac{x_{i, j, r}}{2}\left(\operatorname { l o g } _ { 2 } \left(\frac{\eta_{i, j, r} P_{i, j, r}\left|h_{i, r}\right|^{2}}{\sigma^{2}}\right.\right. \\
& +1)+\log _{2}\left(1+\frac{\left(1-\eta_{i, j, r}\right) P_{i, j, r}\left|h_{j, r}\right|^{2}}{\eta_{i, j, r} P_{i, j, r}\left|h_{j, r}\right|^{2} \sigma^{2}}\right)+\log _{2}(1+ \\
& \left.\left.\frac{\operatorname{Pr}_{i, j, r}\left|f_{i, j, r}\right|^{2}}{\sigma^{2}}\right)\right) .
\end{aligned}
$$

With this, we then face a one to one assignment problem where one channel is assigned to exactly one vehicle pair and only one channel is assigned to each pair. Let us define a matrix 
$Q$ such that:

$$
\boldsymbol{Q}=\left[\begin{array}{cccc}
G(1,1) & G(1,2) & \ldots & G(1, \Lambda / 2) \\
G(2,1) & G(2,2) & \ldots & G(2, \Lambda / 2) \\
\vdots & \vdots & & \vdots \\
G(\Lambda / 2,1) & G(\Lambda / 2,2) & \ldots & G(\Lambda / 2, \Lambda / 2)
\end{array}\right],
$$

where the row and column indexes represent the channel and user pairs, respectively. Hungarian algorithm is a well known technique that gives the optimal solution to such assignment problems [48]. Thus, we employ this technique to assign channels to each vehicle pair ${ }^{4}$. After assigning the channels to the vehicle pairs, the problem of power allocation can be solved using similar steps presented in previous section and are omitted to avoid repetitions. The complexity of this scheme is of order $O\left(\left(\frac{\Lambda}{2}\right)^{3}\right)$. Clearly, $\left(\frac{\Lambda}{2}\right)^{3}<\frac{\Lambda^{3}}{2}$ and, hence, this scheme takes less time to converge.

\section{Simulation RESUlts AND Discussion}

In this section, we present the simulation results for the proposed schemes in Section III and IV. We consider Rayleigh fading channels chosen from independent and identically distributed Gaussian random variables. Unless stated otherwise, we have taken $\Lambda=10, P_{T}=50 \mathrm{~W}, \operatorname{Pmax}_{w}=3 \mathrm{~W}, B=5 \mathrm{~Hz}$, $\psi_{w, r}=0.1 \mathrm{~W}$ and $\sigma^{2}=0.01$ for generating the simulation results. Further, the unit of rate is taken to be bits per second. For the sake of fair comparison, the following schemes are presented:

- Opt: In this scheme we jointly optimize all the parameters $\left(P_{w, e, r}, P r_{e, w, r}, \eta_{w, e, r}, x_{w, e, r}\right)$ as presented in section III.

- Sub-Opt-1: This corresponds to framework where the values of $\operatorname{Pr}_{e, w, r}, \eta_{w, e, r}, x_{w, e, r}$ are optimized in the same fashion as in Opt, whereas, equal values of $P_{w, e, r}$ is allocated to each user pair in the system. This scheme was explained in more detail in section IV-A.

- Sub-Opt-2: In this technique, first the vehicle are paired together according to the pairing scheme proposed in in [49]. Then, Hungarian algorithm is employed for allocated channel to each pair and the values of $P_{w, e, r}, P r_{e, w, r}, \eta_{w, e, r}$ are optimized as proposed in section III. The Sub-Opt 2 scheme is presented in section IV-B.

Fig. 2 demonstrates the impact of increasing the minimum rate requirement of each vehicle on the sum-rate of the proposed schemes. It can be seen that for $R t_{w} \leq 1.2 \mathrm{bps}$, the sumrate remains unchanged for Opt. However, a further increase in $R t_{w}$ results in decreasing the sum-rate because more power is allocated for decoding the data of SV, which then causes power deficiency for decoding the signal of $\mathrm{WV}$ and leads to the decoding errors. Similar trend can be seen for Sub-Opt-1 and Sub-Opt-2. Moreover, the schemes where $P_{w, e, r}$ is calculated according to (29) can vary the powers among pairs. Thus, for an increase in $R t_{w}$, these schemes can allocate more power to the pairs with better channel gains. Hence, for the increasing

${ }^{4}$ For more details on Hungarian algorithm we refer the reader to [50].

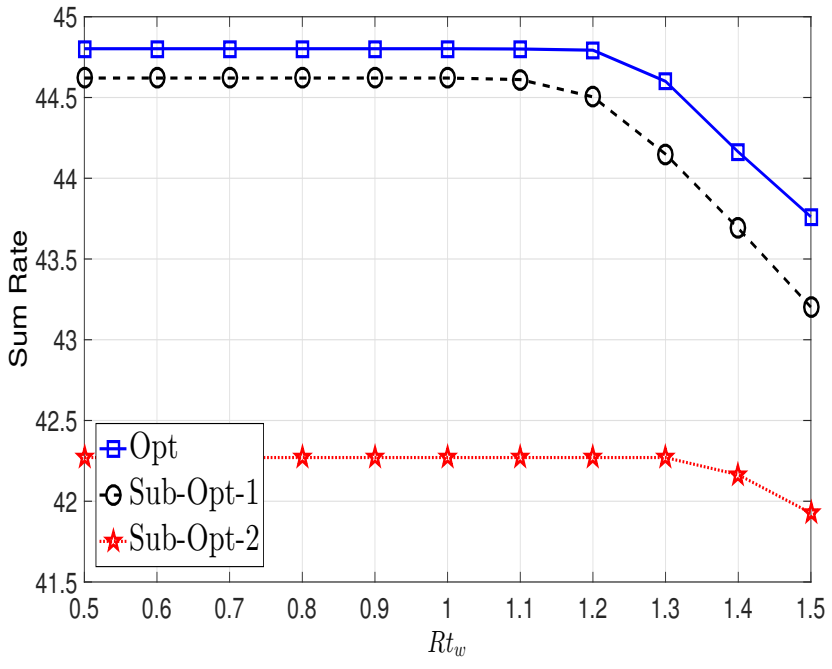

Fig. 2: Effect of increasing $R t_{w}$ on sum-rate.

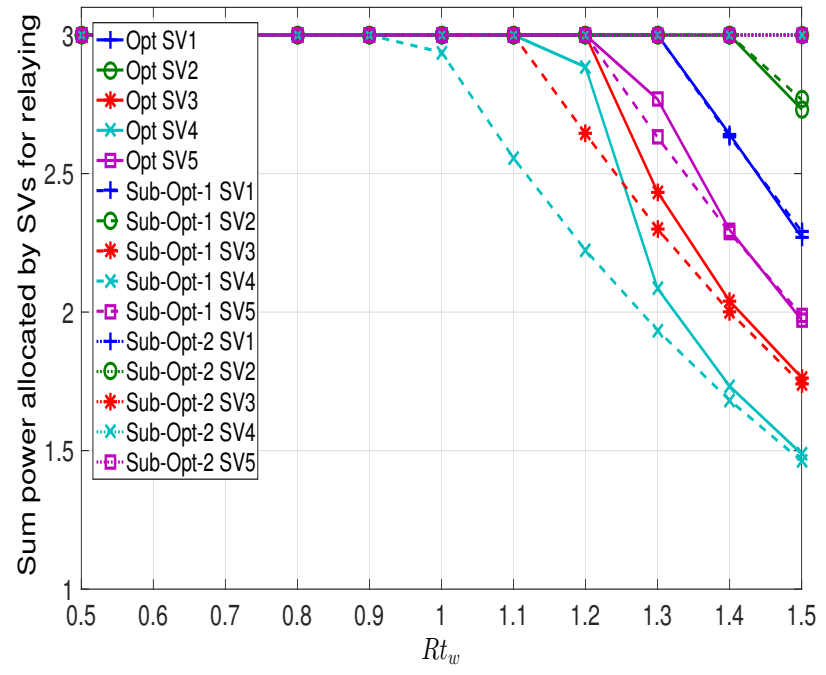

Fig. 3: Impact of increasing $R t_{w}$ on the power used for relaying.

values of $R t_{w}$, the gap between $O p t$ and Sub-Opt-1 increases. On the contrary, the the gap among Sub-Opt-1 and Sub-Opt2 decreases, indicating their convergence at higher values of $R t_{w}$. It is worth mentioning that although for small values of $R T_{w}$ the gap in the rates of Opt and Sub-Opt-1 is small, the main advantage of employing Opt over Sub-Opt-1 lies in the feasibility of the solution. As, for $R t_{w}>1.5$, the rate of some vehicle pairs in the case of Sub-Opt-1 falls below $R t_{w}$ and the power allocated to other vehicle pairs having rate greater than the minimum requirement cannot be reassigned to fulfill the need of each vehicle. Thus, in such cases Sub-Opt-1 fails to provide a feasible solution. However, in the case of $O p t$, when the rate of a vehicle falls below $R t_{w}$, the power allocated to other vehicle pairs is reduced and the extra power is allocated to the respective vehicle pair. This results in satisfying the requirement of every user in the system. Moreover, it can be seen from the figure that Opt outperforms theSub-Opt-1 and Sub-Opt-2 for all values of $R t_{w}$. 


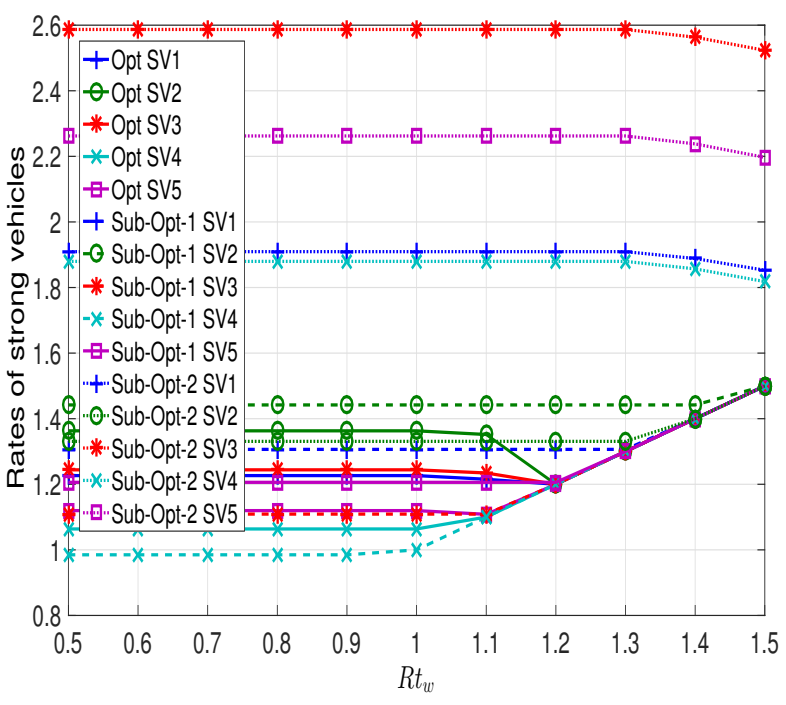

Fig. 4: Rate of SVs versus $R t_{w}$.

The impact of different values of $R t_{w}$ on $P r_{e, w, r}$ for each scheme is illustrated in Fig. 3. Initially increasing $R t_{w}$ does not affect $P r_{e, w, r}$, and each SV relays the data with full available power. However, further increasing the value of $R t_{w}$ depletes the power for information decoding at the SV. Thus, the power allocated for relaying the message decreases. It can be seen that in $O p t$, the SVs are able to relay with full power even at higher values of $R t_{w}$ as compared to Sub-Opt-1. The Opt scheme is capable of varying the available power among vehicle pairs, and hence power is subtracted from the vehicle pairs where SVs are relaying with full available power and have a rate greater than $R t_{w}$. This power is added to the pairs where the rate of $\mathrm{SV}$ is equal to $R t_{w}$, thus power ratio can be adjusted to relay with more power. This adjustment of power results in all SV relaying with full available power. Moreover, when $R t_{w}$ is increased to the extent where the SVs in $O p t$ cannot relay with full power and $P_{w, e, r}$ cannot be adjusted. Then, $P_{w, e, r}$ is allocated so that the sum-rate can be maximized by allocating more $P_{w, e, r}$ to the pairs with better channels. Whereas, Sub-Opt-1 is incapable of adjusting powers among vehicle pairs which makes the sum-rate of $O p t$ greater than Sub-Opt-1. All SVs in Sub-Opt-2 are capable of relaying with full power even at higher values of $R t_{w}$. Apparently, the performance of Sub-Opt-2 must be better than the other schemes. However, we note that in Sub-Opt-2, the user pairing does not account for the channel from the SV to WV. Thus, these channels are weaker compared to those selected in $O p t$ and Sub-Opt-1. Hence, even with full transmit power, the rate provided by relaying in Sub-Opt-2 is far less than that in other schemes. This results in less overall rate, which is evident from the results of Fig. 3.

Fig. 4 shows the effect of increasing $R t_{w}$ on the rates of $\mathrm{SVs}$ for the proposed schemes. It is worth noting that for increasing values of $R t_{w}$, at first there is no change in the rate of any user because the rate of each SV is already greater than $R t_{w}$. Interestingly, we observe that in the schemes where $P_{w, e, r}$ is updated using (29), when the rate of an SV is below the minimum requirement, the power allocated to the specific

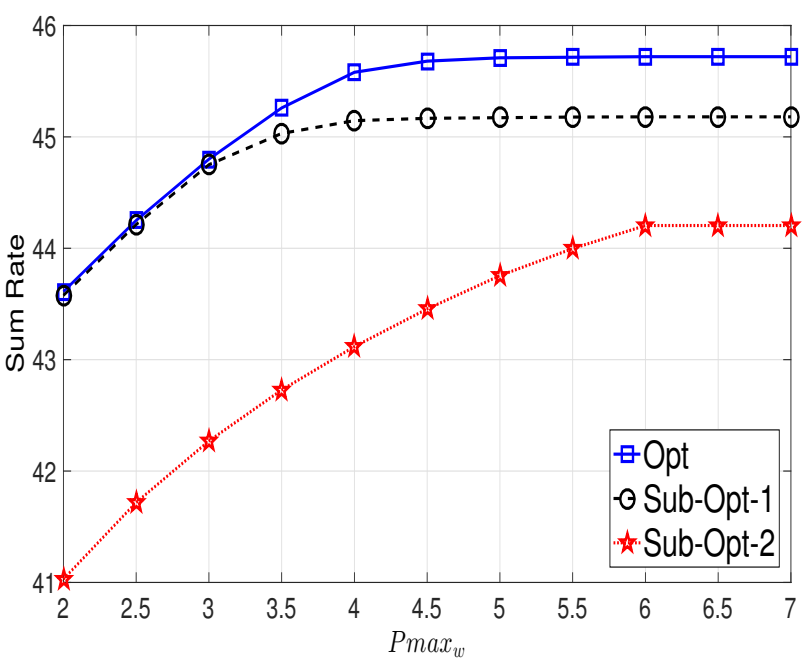

Fig. 5: Effect of $P \max _{w}$ on the sum-rate of the system, where $R t_{w}=$ $1 \mathrm{bps} / \mathrm{Hz}$.

user is increased to fulfill the threshold requirements. While the power allocated to the other SVs having rate greater than $R t_{w}$ decreases. This is because, if for increasing the rate of an SV we adjust $\eta_{w, e, r}$ the SV might not be able to relay the decoded data with full power. Hence, Opt and Sub-Opt2 schemes adjust $P_{w, e, r}$ by decreasing power of strong pairs that are relaying with full power. This additional power is added to the transmissions of vehicles where the rates of the SVs needs to be increased. In contrast, for the case of SubOpt-1, the value of $P_{w, e, r}$ for each pair of vehicle is fixed. Thus, the power allocation to the pairs cannot be adjusted and consequently, a decrease in the allocated power for relaying is observed. In this case, the rates of every other SV remains unchanged. For all the proposed schemes, when the rate of each SV becomes equal to $R t_{w}$, a further increase in $R t_{w}$ is required to adjust $\eta_{w, e, r}$, which reduces the power allocated for the signal of WV, causing the power allocated for relaying to decrease. Due to this reason, the overall achievable rate of every scheme suffers in Fig 2.

Fig. 5 shows the impact of $P \max _{w}$ on the sum-rate of each scheme. For all the remaining results we consider $R t_{w}=$ $1 \mathrm{bps} / \mathrm{Hz}$. It can be seen that the performance of $O p t$ is better than the remaining two schemes for all values of $\operatorname{Pmax}_{w}$. In general, an increase in $\operatorname{Pmax}_{w}$ enhances the sum-rate of each scheme. After a point the sum-rate of each scheme is not affected by increasing $\operatorname{Pmax}_{w}$ because the achievable rate of each SV in the system becomes equal to $R t_{w}$. Hence, changing $\eta_{w, e, r}$ and/ or $P_{w, e, r}$ to increase the power allocated for the signals of the WVs will result in violating $R t_{w}$ of the SVs. Both $O p t$ and $S u b-O p t-2$ can vary $P_{w, e, r}$ to take the advantage of the increasing $\operatorname{Pmax}_{w}$ by allocating more power to the pairs that have better channel gain between SV and WV. Thus, with an increase in $\operatorname{Pmax}_{w}$, the gap between $O p t$ and $S u b$ Opt-1 increases, whereas, the difference between the sum-rate of Sub-Opt-1 and Sub-Opt-2 decreases.

The impact of increasing $\operatorname{Pmax}_{w}$ on the sum power allocated for relaying by each scheme is illustrated in Fig 6 . 


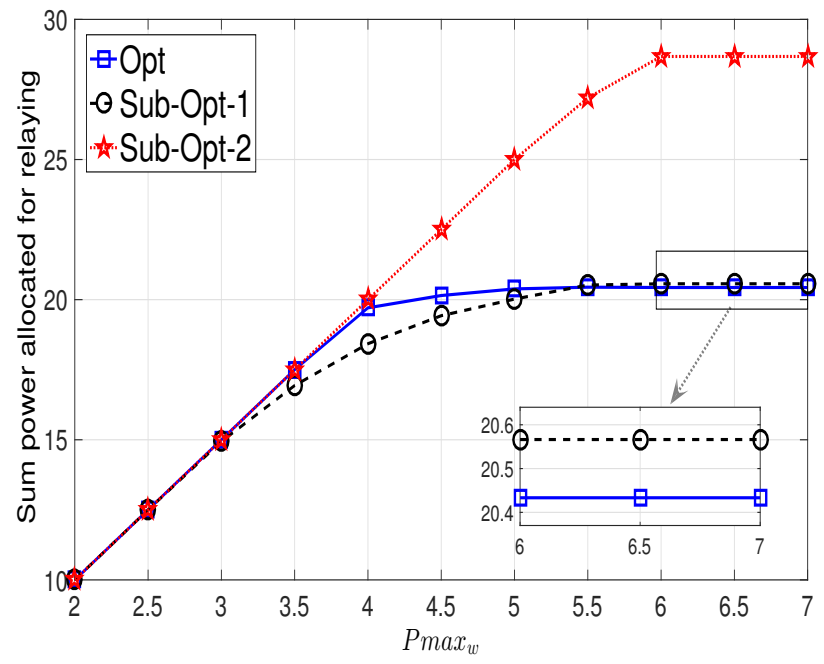

Fig. 6: Total power used for relaying versus $P \max _{w}$, where $R t_{w}=$ $1 \mathrm{bps} / \mathrm{Hz}$.

In general, the total power allocated for relaying increases with increasing values of $\operatorname{Pmax}_{w}$ while becoming constant at higher values. For higher values of $\operatorname{Pmax}_{w}$, although the power used for relaying by Sub-Opt-2 is greater than that of other schemes, the achieved sum-rate is the lowest. This is because the vehicle pairing in Sub-Opt-2 just accounts for the response of channels from the BS to the vehicle. Whereas, in Opt and Sub-Opt-1 the channel gains from SVs to WVs are also considered. In Sub-Opt-1, initially each SV relays with full power available. However, for $\operatorname{Pmax}_{w}>3$, the rate of a few SVs approaches $R t_{w}$. Thus, $P_{w, e, r}$ cannot be varied, so, power allocated for the signal of $\mathrm{WV}$ does not change. Consequently, the SVs cannot invest more power to relay the data of WVs. For the SVs having a rate greater than $R t_{w}, \eta_{w, e, r}$ is adjusted to increase the power of the WV signal. The increase in $\operatorname{Pmax}_{w}$ makes the rate of more SV equal to $R t_{w}$, which explains the decrease in the slope of Sub-Opt-1. Moreover, for $\operatorname{Pmax}_{w}>5.5$ the power allocated by Sub-Opt-1 surpasses the Opt. Yet, it was seen from the results of Fig. 5 that the sum rate of $O p t$ is greater. This is because, for the case of $O P T$, the available power at the BS is distributed between the vehicle pairs such that the overall rate can be maximized. However, the same is not true in the case of Sub-Opt-1, wherein, the available power is distributed equally between the pairs.

Fig. 7 shows the effect of changing $P_{T}$ on sum-rate of the proposed schemes. The rates offered by each scheme increase with $P_{T}$, whereas, the gap between $O P T$ and $S u b$ Opt-1 decrease. This is because at lower values of $P_{T}$, some SVs have rate equal to $R t_{T}$ that are unable to use full available power for relaying. When $P_{T}$ is increased, the power used for transmitting the signal of WV increases. Hence, the corresponding SV can relay the data with more power. It can be seen that when all the SV in $O P T$ and Sub-Opt-1 are relaying with full available power, there is a small difference between the sum-rate of both schemes.

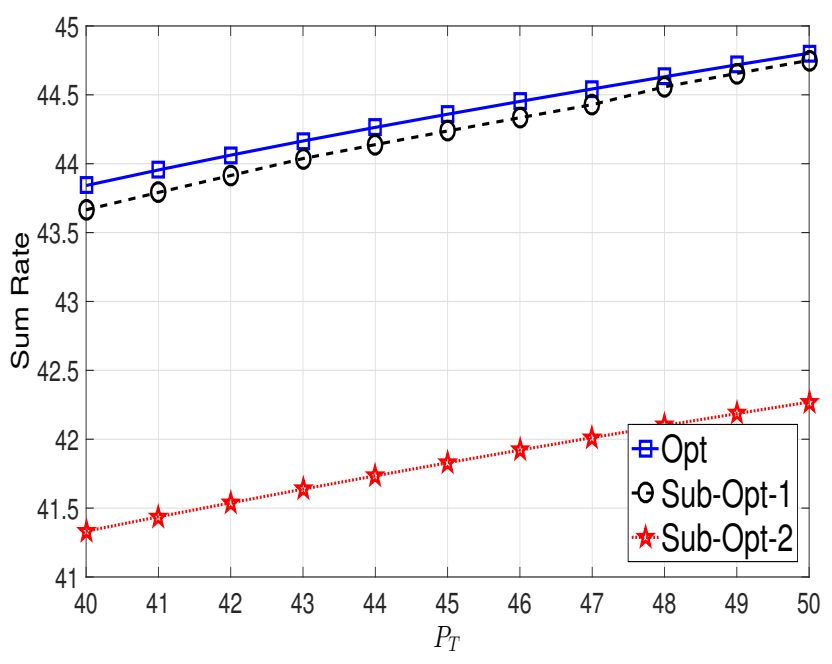

Fig. 7: Impact of $P_{T}$ on the sum-rate.

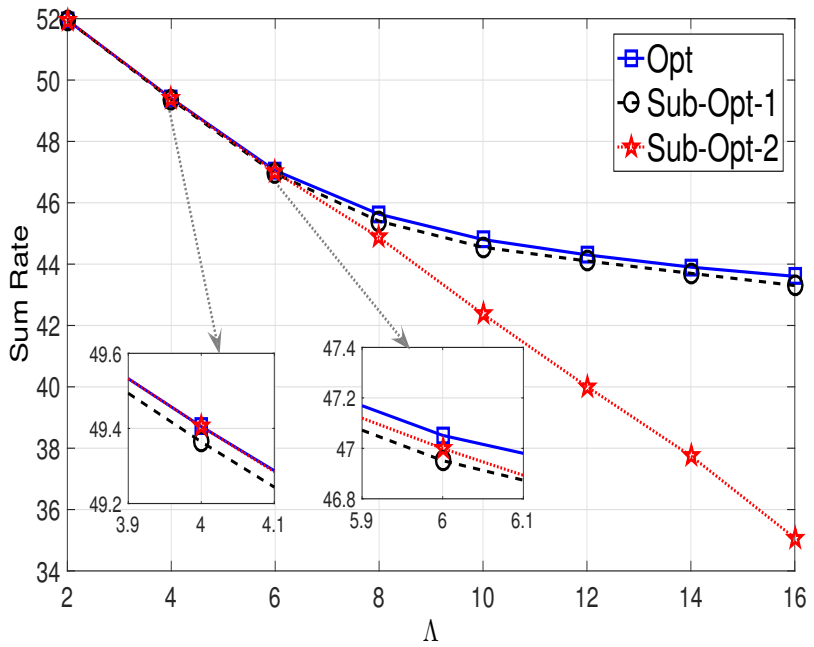

Fig. 8: Sum-rate versus number of vehicles in the system.

The effect of increasing the value of $\Lambda$ on the sum-rate of each scheme is presented in Fig. 8. It may be noted that the rate of each scheme decreases with the increase in $\Lambda$ because of the reduction in the bandwidth of each channel. As the value of $\Lambda$ is increased, it can be seen that the decrease in the sum-rate of Sub-Opt-2 is more rapid as compared to OPT and SubOpt-1. This is mainly because the later schemes intelligently pair vehicles together to compensate for the decrease in rate. It is obvious that for two vehicles the rate of each scheme will be the same. However, it is interesting to note that for 4 and 6 users, Sub-Opt-2 performs better than Sub-Opt-1. This is because for lower values of $\Lambda$, there is a high probability that the vehicle pairing of Sub-Opt-2 will be the same as that of the $O P T$. Moreover, it is worth noting that $O P T$ outperforms the other schemes because the sum-rate provided by $O P T$ is always greater than or equal to that of other schemes. 


\section{CONCLUSION}

This paper has provided a new optimization framework for NOMA-enabled cooperative vehicular networks. Specifically, a joint optimization problem of power loading, vehicle pairing, and channel allocation has been provided to maximize the sum rate of the system. The optimization problem has also been accounted for the individual rate requirement, battery capacity for relaying at each vehicle, total source power, and a reasonable gap between the received powers to facilitate NOMA transmission. Efficient solutions have been derived using dual theory and KKT conditions, where the dual variables have been iteratively updated using the sub-gradient method. The simulation results demonstrate that the proposed joint optimization scheme outperforms the other benchmark cooperative vehicular schemes.

\section{APPENDIX \\ CONVEXITy ANALYSIS OF PROBLEM P2}

Here we discuss the convexity of the problem in P2. First we calculate the Hessian of the objective function as given below.

$$
\text { Hessian }_{1}=\left[\begin{array}{ccc}
\bar{\Omega}_{1} & \bar{\Psi}_{1} & 0 \\
\bar{\chi}_{1} & \bar{\vartheta}_{1} & 0 \\
0 & 0 & \bar{\xi}_{1}
\end{array}\right],
$$

where:

$$
\begin{gathered}
\bar{\Omega}_{1}=\frac{\left(\left|h_{e, r}\right|^{2}-\left|h_{w, r}\right|^{2}\right) P_{w, e, r}^{2} \sigma^{2} a_{1}}{\left(\eta_{w, e, r}\left|h_{e, r}\right|^{2} P_{w, e, r}+\sigma^{2}\right)^{2}\left(\eta_{w, e, r}\left|h_{w, r}\right|^{2} P_{w, e, r}+\sigma^{2}\right)^{2}}, \\
a_{1}=\left(2 \eta_{w, e, r}\left|h_{e, r}\right|^{2}\left|h_{w, r}\right|^{2} P_{w, e, r}+\left(\left|h_{e, r}\right|^{2}+\left|h_{w, r}\right|^{2}\right) \sigma^{2}\right), \\
\bar{\Psi}_{1}=\frac{\left(\left|h_{e, r}\right|^{2}-\left|h_{w, r}\right|^{2}\right) \sigma^{2}\left(\eta_{w, e, r}^{2}\left|h_{e, r}\right|^{2}\left|h_{w, r}\right|^{2} P_{w, e, r}^{2}-\sigma^{2}\right)}{\left(\eta_{w, e, r}^{2}\left|h_{e, r}\right|^{2} P_{w, e, r}+\sigma^{2}\right)^{2}\left(\eta_{w, e, r}\left|h_{w, r}\right|^{2} P_{w, e, r}+\sigma^{2}\right)^{2}}, \\
-\frac{\left(a_{w, e, r}-1\right)\left|h_{e, r}\right|^{4} \sigma^{2}\left(\sigma^{2}+\eta_{w, e, r}\left(2\left|h_{e, r}\right|^{2} P_{w, e, r}+\sigma^{2}\right)\right)}{\left(\left|h_{e, r}\right|^{2} P_{w, e, r}+\sigma^{2}\right)^{2}\left(\eta_{w, e, r}\left|h_{e, r}\right|^{2} P_{w, e, r}+\sigma^{2}\right)^{2}} \\
\bar{\vartheta}_{1}=\frac{\eta_{w, e, r}^{2}\left|h_{w, r}\right|^{4}}{\left(1+\frac{\eta_{w, e, r}\left|h_{w, r}\right|^{2} P_{w, e, r}}{\sigma^{4}} \sigma^{4}\right)}, \\
\bar{\xi}_{1}=-\frac{\left|f_{e, w, r}\right|^{4}}{\left(1+\left(\frac{\left|f_{e, w, r}\right|^{2} P r_{e, w, r}}{\sigma^{2}}\right)^{2} \sigma^{4}\right)}, \bar{\chi}_{1}=\bar{\Psi}_{1},
\end{gathered}
$$

Applying row operations to convert the Hessian 1 into an upper triangular matrix we get:

$$
\text { Hessian }_{2}=\left[\begin{array}{ccc}
\bar{\Omega}_{1} & \bar{\Psi}_{1} & 0 \\
0 & \bar{\vartheta}_{2} & 0 \\
0 & 0 & \bar{\xi}_{1}
\end{array}\right],
$$

for $\bar{\vartheta}_{2}$ as given below:

$$
\bar{\vartheta}_{2}=\frac{a_{3}}{P_{w, e, r}^{2}\left(\left|h_{e, r}\right|^{2} P_{w, e, r}+\sigma^{2}\right)^{2}\left(\eta_{w, e, r}\left|h_{e, r}\right|^{2} P_{w, e, r}+\sigma^{2}\right) a_{2}}
$$

where:

$$
\begin{aligned}
& a_{2}=\left(\eta_{w, e, r}\left|h_{w, r}\right|^{2} P_{w, e, r}+\sigma^{2}\right)\left(2 \eta_{w, e, r}\left|h_{e, r}\right|^{2}\left|h_{w, r}\right|^{2}\right. \\
& \left.P_{w, e, r}+\left(\left|h_{e, r}\right|^{2}+\left|h_{w, r}\right|^{2}\right) \sigma^{2}\right), \\
& a_{3}=-2 \eta_{w, e, r}^{3}\left|h_{e, r}\right|^{8}\left|h_{w, r}\right|^{4} P_{w, e, r}^{5}-6 \eta_{w, e, r}^{2}\left|h_{e, r}\right|^{6}\left|h_{w, r}\right|^{4} \\
& P_{w, e, r}^{4} \sigma^{2}+\left(\left|h_{e, r}\right|^{6}\left|h_{w, r}\right|^{2} P_{w, e, r}^{3}\left(-4 \eta_{w, e, r}+6 \eta_{w, e, r}^{2}\right)-\right. \\
& \left.\left|h_{e, r}\right|^{4}\left|h_{w, r}\right|^{4} P_{w, e, r}^{3} 6 \eta_{w, e, r}^{2}\right) \sigma^{4}+\left(\left(2\left|h_{e, r}\right|^{6}\left(\eta_{w, e, r}-1\right)+\right.\right. \\
& \left.\left.3 \eta_{w, e, r}^{2}\left(\left|h_{e, r}\right|^{4}\left|h_{w, r}\right|^{2}-\left|h_{e, r}\right|^{2}\left|h_{w, r}\right|^{4}\right)\right) P_{w, e, r}^{2}\right) \sigma^{6}+(-2 \\
& \left.\left|h_{e, r}\right|^{4}+\eta_{w, e, r}\left(\left|h_{e, r}\right|^{4}-\left|h_{w, r}\right|^{4}\right)\right) P_{w, e, r} \sigma^{8}+\left(\sigma^{2}-\left|h_{w, r}\right|^{4}\right. \\
& \left.\eta_{w, e, r}\right) 2 P_{w, e, r}\left|h_{e, r}\right|^{2}\left|h_{w, r}\right|^{2} \sigma^{6}+\left|h_{w, r}\right|^{2} \sigma^{4}\left(\sigma^{6}-2 \eta_{w, e, r}\right. \\
& \left.\left|h_{e, r}\right|^{4}\left|h_{w, r}\right|^{4} P_{w, e, r}^{3}\right)-\left|h_{e, r}\right|^{2} \sigma^{10} .
\end{aligned}
$$

Under the considered system we have $\left|h_{w, r}\right|^{2}>\left|h_{e, r}\right|^{2}$ and $\eta_{w, e, r}<\frac{1}{2}$. Thus, for $\frac{\sigma^{2}}{\eta_{w, e, r}}<\left|h_{e, r}\right|$, we get $\bar{\Omega}_{1}<0, \bar{\vartheta}_{2}<0$ and $\bar{\xi}_{1}<0$. The eigenvalues of a triangular matrix are the elements of the principal diagonal, we can see that all the eigenvalues of $\mathrm{Hessian}_{2}$ are negative. Thus, the Hessian matrix is negative definite and the objective function in P2 is strictly concave. The constraints in (14) and (15) are linear in $P_{w, e, r}$ and $P r_{w, e, r}$, respectively. However, the Hessian of (16) has eigenvalues of $\{-2,2\}$, and hence, the constraint is nonconvex (neither convex nor concave). The fourth constraint is strictly concave and has following eigenvalue:

$$
\begin{aligned}
& E V_{1}=-\frac{\left|h_{e, r}\right|^{4} P_{w, e, r}^{2}}{\left(\eta_{w, e, r}\left|h_{e, r}\right|^{2} P_{w, e, r}+\sigma^{2}\right)^{2}}, \\
& E V_{2}=\frac{-\eta_{w, e, r}\left|h_{e, r}\right|^{2} P_{w, e, r}+\sigma^{2}}{P_{w, e, r}^{2}\left(\eta_{w, e, r}\left|h_{e, r}\right|^{2} P_{w, e, r}+\sigma^{2}\right)} .
\end{aligned}
$$

Further, (18) and (19) are both convex in $\eta_{w, e, r}$ and concave in $P_{w, e, r}$, whereas, for $P r_{e, w, r}$ the constraints in (18) and (19) are concave and convex, respectively.

\section{REFERENCES}

[1] J. He, K. Yang and H. -H. Chen, "6G Cellular networks and connected autonomous vehicles," IEEE Network, pp. 1-1, 2020, doi: 10.1109/MNET.011.2000541.

[2] W. U. Khan, X. Li, A. Ihsan, M. A. Khan, V. G. Menon and M. Ahmed, "NOMA-enabled optimization framework for next-generation small-cell IoV networks under imperfect SIC decoding," IEEE Transactions on Intelligent Transportation Systems, pp. 1-1, 2021, doi: 10.1109/TITS.2021.3091402.

[3] S. Chen, J. Hu, Y. Shi, and L. Zhao, "Technologies, standards and applications of LTE-V2X for vehicular networks," Telecommun. Science, vol. 34, no. 4, pp. 1-11, 2018.

[4] F. Jameel, W. U. Khan, N. Kumar and R. Jntti, "Efficient powersplitting and resource allocation for cellular V2X communications," IEEE Transactions on Intelligent Transportation Systems, vol. 22, no. 6, pp. 3547-3556, June 2021.

[5] J. Luo, J. Tang, D. K. C. So, G. Chen, K. Cumanan and J. A. Chambers, "A Deep learning-based approach to power minimization in multicarrier NOMA with SWIPT," IEEE Access, vol. 7, pp. 17450-17460, 2019.

[6] W.U. Khan, X. Li, A. Ihsan, Z. Ali, B. M Elhalawany, G. A. S. Sidhu, "Energy efficiency maximization for beyond 5G NOMAenabled heterogeneous networks", Peer-to-Peer Netw. Appl., 2021, https://doi.org/10.1007/s12083-021-01176-5.

[7] Y. Sun, Z. Ding, X. Dai, K. Navaie and D. K. C. So, "Performance of downlink NOMA in vehicular communication networks: An analysis based on poisson line cox point process," IEEE Trans. on Veh. Technol., vol. 69, no. 11, pp. 14001-14006, Nov. 2020. 
[8] G. Gui, H. Sari and E. Biglieri, "A new definition of fairness for nonorthogonal multiple access," IEEE Communications Letters, vol. 23, no. 7, pp. 1267-1271, July 2019.

[9] W. U. Khan, J. Liu, F. Jameel, V. Sharma, R. Jantti and Z. Han, "Spectral efficiency optimization for next generation NOMA-enabled IoT networks," IEEE Transactions on Vehicular Technology, vol. 69 no. 12, pp. 15284-15297, Dec. 2020.

[10] Y. Wu, E. Attang and G. E. Atkin, "Low complexity NOMA system with combined constellations," IEEE Wireless Communications Letters, vol. 8, no. 5, pp. 1308-1311, Oct. 2019.

[11] S. Mao, S. Leng, J. Hu and K. Yang, "Power minimization resource allocation for underlay MISO-NOMA SWIPT systems," IEEE Access, vol. 7, pp. 17247-17255, 2019.

[12] W. U. Khan, F. Jameel, T. Ristaniemi, S. Khan, G. A. S. Sidhu, J. Liu "Joint spectral and energy efficiency optimization for downlink NOMA networks," IEEE Trans. Cognitive Commun. Netw., vol. 6, no. 2, pp. 645-656, Jun. 2020.

[13] W. U. Khan, X. Li, M. Zeng and O. A. Dobre, "Backscatter-enabled NOMA for future $6 \mathrm{G}$ systems: A new optimization framework under imperfect SIC," IEEE Communications Letters, vol. 25, no. 5, pp. 1669-1672, May 2021.

[14] Y. Liu, Z. Qin, M. Elkashlan, Z. Ding, A. Nallanathan and L. Hanzo, "Nonorthogonal multiple access for 5G and beyond," Proceed. of the IEEE, vol. 105, no. 12, pp. 2347-2381, 2017.

[15] L. Wei, Y. Chen, D. Zheng and B. Jiao, "Secure performance analysis and optimization for FD-NOMA vehicular communications," China Commun., vol. 17, no. 11, pp. 29-41, Nov. 2020.

[16] W. U. Khan, F. Jameel, M. A. Jamshed, H. Pervaiz, S. Khan, J. Liu, "Efficient power allocation for NOMA-enabled IoT networks in 6G era," Physical Communication, vol. 39, 101043, 2020, https://doi.org/10.1016/j.phycom.2020.101043.

[17] Y. Xu, C. Yang, M. Hua and W. Zhou, "Deep deterministic policy gradient (DDPG)-based resource allocation scheme for NOMA vehicular communications," IEEE Access, vol. 8, pp. 18797-18807, 2020.

[18] W. U. Khan, F. Jameel, X. Li, M. Bilal and T. A. Tsiftsis, "Joint spectrum and energy optimization of NOMA-enabled small-cell networks with QoS guarantee," IEEE Transactions on Vehicular Technology, vol. 70, no. 8, pp. 8337-8342, Aug. 2021.

[19] D.-T. Do, A.-T. Le, T.-A. Hoang, and B. M. Lee, "Cognitive radioassisted NOMA broadcasting for $5 \mathrm{G}$ cellular V2X communications: Model of roadside unit selection and SWIPT," Sensors, vol. 20, no. 6, p. 1786,2020

[20] N. Jaiswal and N. Purohit, "Performance of downlink NOMA-enabled vehicular communications over double Rayleigh fading channels," IET Communications, vol. 14, no. 20, pp. 3652-3660, 2020.

[21] D. T. Do, M. S. V. Nguyen, A. T. Le, K. M. Rabie, and J. Zhang, "Joint full-duplex and roadside unit selection for NOMA-enabled V2X communications: ergodic rate performance," IEEE Access, vol. 8, pp. 140348-140360, 2020

[22] D. Zhang, Y. Liu, L. Dai, A. K. Bashir, A. Nallanathan and B. Shim, "Performance analysis of decentralized V2X system with FD-NOMA," IEEE 90th Vehicular Technology Conference (VTC2019-Fall), Honolulu, HI, USA, 2019, pp. 1-6, doi: 10.1109/VTCFall.2019.8891360.

[23] D. Zhang, Y. Liu, L. Dai, A. K. Bashir, A. Nallanathan and B. Shim, "Performance analysis of FD-NOMA-based decentralized V2X systems," IEEE Transactions on Communications, vol. 67, no. 7, pp. 5024-5036, July 2019.

[24] W. U. Khan, M. A. Javed, T. N. Nguyen, S. Khan, B. M. Elhalawany, "Energy-efficient resource allocation for $6 \mathrm{G}$ backscatterenabled NOMA IoV networks," 2021, TechRxiv. Preprint, https://doi.org/10.36227/techrxiv.15133902.v1.

[25] B. Wang, R. Zhang, C. Chen, X. Cheng, L. Yang, and Y. Jin, "Interference hypergraph-based 3D matching resource allocation protocol for NOMA-V2X networks," IEEE Access, vol. 7, pp. 90789-90800, 2019.

[26] O. Abbasi, H. Yanikomeroglu, A. Ebrahimi, and N. M. Yamchi, "Trajectory design and power allocation for drone-assisted NR-V2X network with dynamic NOMA/OMA," IEEE Transactions on Wireless Communications, vol. 19, no. 11, pp. 7153-7168, Nov. 2020.

[27] H. Zheng, H. Li, S. Hou, and Z. Song, "Joint resource allocation with weighted max-min fairness for NOMA-enabled V2X communications," IEEE Access, vol. 6, pp. 65449-65462, 2018.

[28] D. Zhang, Y. Liu, L. Dai, A. K. Bashir, A. Nallanathan, and B. Shim, "Performance analysis of FD-NOMA-based decentralized V2X systems," IEEE Transactions on Communications, vol. 67, no. 7, pp. 5024-5036, Jul. 2019

[29] H. Xiao, Y. Chen, S. Ouyang, and A. T. Chronopoulos, "Power control for clustering car-following V2X communication system with nonorthogonal multiple access," IEEE Access, vol. 7, pp. 68160-68171, 2019.

[30] B. Di, L. Song, Y. Li, and G. Y. Li, "Non-orthogonal multiple access for high-reliable and low-latency V2X communications in 5G systems," IEEE Journal on Selected Areas in Communications, vol. 35, no. 10, pp. 2383-2397, Oct. 2017.

[31] S. Guo and X. Zhou, "Robust resource allocation with imperfect channel estimation in NOMA-based heterogeneous vehicular networks," IEEE Transactions on Communications, vol. 67, no. 3, pp. 2321-2332, March 2019

[32] W. U. Khan, F. Jameel, G. A. S. Sidhu, M. Ahmed, X. Li, and R Jntti, "Multiobjective optimization of uplink NOMA-enabled vehicletoinfrastructure communication," IEEE Access, vol. 8, pp. 84467-84478, 2020.

[33] A. Ihsan, W. Chen, S. Zhang and S. Xu, "Energy-efficient NOMA multicasting system for beyond $5 \mathrm{G}$ cellular V2X communications with imperfect CSI," IEEE Transactions on Intelligent Transportation Systems, pp. 1-1, 2021, doi: 10.1109/TITS.2021.3095437.

[34] L. Wei, Y. Chen, D. Zheng and B. Jiao, "Secure performance analysis and optimization for FD-NOMA vehicular communications," China Communications, vol. 17, no. 11, pp. 29-41, Nov. 2020

[35] W. U. Khan, F. Jameel, N. Kumar, R. Jantti, M. Guizani, "Backscatterenabled efficient V2X communication with non-orthogonal multiple access", IEEE Trans. Veh. Technol., vol. 70, no. 2, pp. 1724-1735, Feb. 2021.

[36] Y. Saito, Y. Kishiyama, A. Benjebbour, T. Nakamura and A. Li, "Nonorthogonal multiple access (NOMA) for cellular future radio access," in Proc. of IEEE Veh. Techn. Conf., pp. 1-5, Dresden, Germany, Jun. 2013.

[37] Y. Liu, H. Zhang, K. Long, A. Nallanathan, and V. C. Leung, Energyefficient subchannel matching and power allocation in NOMA autonomous driving vehicular networks, IEEE Wireless Communications, vol. 26, no. 4, pp. 88-93, Aug. 2019.

[38] W. U. Khan, Z. Yu, S. Yu1, G. A. S. Sidhu and J. Liu, Efficient power allocation in downlink multi-cell multi-user NOMA networks, IET Communications, Vol. 13, No. 4, pp. 396-402, 2019.

[39] H. Zhang, Y. Ma, D. Yuan and H. Chen, "Quality-of-Service Driven Power and Sub-Carrier Allocation Policy for Vehicular Communication Networks," IEEE J. on Sel. Areas in Commun., vol. 29, no. 1, pp. $197-$ 206, Jan. 2011

[40] H. Ilhan, I. Altunbas and M. Uysal, "Cooperative diversity for relayassisted inter-vehicular communication," VTC Spring 2008 - IEEE Vehicular Technology Conference, Marina Bay, Singapore, 2008, pp. 605-609.

[41] Z. Mo, W. Su, S. Batalama and J. D. Matyjas, "Cooperative communication protocol designs based on optimum power and time allocation," IEEE Trans. on Wireless Commun., vol. 13, no. 8, pp. 4283-4296, Aug. 2014

[42] E. Zimmermann, P. Herhold and G. Fettweis, "On the performance of cooperative relaying protocols in wireless networks," European Trans. on Telecom., vol. 16, no. 1, pp. 5-16, Feb. 2005.

[43] P. Herhold, E. Zimmermann, G. Fettweis, "Cooperative multi-hop transmission in wireless networks," Computer Networks, vol. 49, no. 3, pp. 299-324, Oct. 2005.

[44] Z. Ali, G. A. S. Sidhu, S. Zhang, L. Xing and F. Gao, " Achieving green transmission with energy harvesting based cooperative communication," IEEE Access, vol. 6, pp. 27507-27517, 2018.

[45] S. Boyd and L. Vandenberghe, Convex Optimization. Cambridge University Press, 2004.

[46] C. Liu, X. Qin, S. Zhang and W. Zhou, "Proportional-fair downlink resource allocation in OFDMA-based relay networks," J. Commun. and Networks, vol. 13, no. 6, pp. 633-638, 2011.

[47] S. Boyd and A. Mutapcic, "Subgradient methods," notes for EE364, Standford University, Winter 2006-07.

[48] Z. Ali, G. A. S. Sidhu, M. Waqas and F. Gao, "Optimizing multi-user CR transmission under RF energy harvesting," in Physical Commun., vol. 32, pp. 209-216, 2019.

[49] M. S. Ali, H. Tabassum and E. Hossain, ”Dynamic user clustering and power allocation for uplink and downlink non-orthogonal multiple access (NOMA) systems," IEEE Access, vol. 4, pp. 6325-6343, 2016.

[50] H. Khun, "The hungarian method for the assignment problems," in Naval Research Logistics, Quarterly 2, pp. 83-97, 1955. 